\title{
Image Compression, ECG Data Compression and Image Encryption by Binary Versions of Discrete Cosine Transform, Haar Transform and Discrete Hartley Transform
}

\author{
P. Ramesh ${ }^{1}$, B. I. Neelgar ${ }^{2}$ and B. M. S. Sreenivasa Rao ${ }^{3}$ \\ $1 P G$ Scholar, \\ ${ }^{1}$ Department of Electronics and Communication Engineering \\ GMR Institute of Technology \\ Rajam, Andhra Pradesh \\ ${ }^{2}$ Professor\& HOD, Department of Electronics and Communication Engineering \\ GMR Institute of Technology \\ Rajam, Andhra Pradesh \\ ${ }^{3}$ Assistant Professor, Department of Electronics and Communication Engineering \\ GMR Institute of Technology \\ Rajam, Andhra Pradesh \\ ${ }^{1}$ rammi490@gmail.com, ${ }^{2}$ hod_ece@gmrit.org, ${ }^{3}$ muraliauece@gmail.com
}

\begin{abstract}
In this paper, it is proposed to implement the image compression, encryption and ECG data compression using by binary description of Discrete Cosine Transform (DCT), Binary Haar Transform and Discrete Hartley Transform (DHT). In this Binary Discrete Cosine Transform (Binary DCT), Binary Haar Transform and Binary Discrete Hartley Transforms (Binary DHT) are developed using the Walsh Hadamard transform (WHT). The resulting transform nearly exact the underlying transform very well, while maintaining all the advantages and properties of WHT. The Binary DCT is a well known sequency ordered Walsh Hadamard Transform (WHT), where as the Binary DHT can be considered as a new Hartley ordered WHT. Specifically, the properties of the proposed Hartley ordering are discussed and a shift copy scheme is proposed for a simple and direct generation of the Hartley ordering functions.
\end{abstract}

Keywords: Walsh Hadamard Transform (WHT), Discrete Cosine Transform (DCT), Discrete Hartley Transform (DHT), Binary Versions of DCT and DHT, Haar Transform and Binary Haar Transform (Binary Haar Transform)

\section{Introduction}

There are variations of discrete orthogonal transforms such as, Walsh Hadamard Transform (WHT), Discrete Cosine Transform (DCT), Discrete Hartley Transform (DHT), Discrete Fourier Transform (DFT), and Harr Transforms, which have different applications in signal processing, image compressing, data compressing and image encryption [10]. DCT is a cut-down form of the Fast Fourier Transform or Fourier Transform. In image compression most of the signal energy lies at low frequencies by DCT, then the DCT is good for its high energy compaction capacity. The WHT have only +1 and -1 and the Walsh functions are orthogonal [6]. In general, the Walsh function can be generated using Hadamard matrix. The DHT takes advantages of symmetry in the DFT of a real numbers or sequences. The Haar transform uses Haar function for its basis. The Haar function is a compact, dyadic, orthonormal and rectangular pair [12]. Compare to the Fourier Transform basis function which only differs in frequency, the Haar function changes in both scale and position. 


\section{Walsh Hadamard Transform (WHT)}

Walsh Hadamard Transform $W_{h}(x)$ for $\mathrm{N}$ length of sequence, In this case positive integer $u$ and it is $u=\log _{2} N$. The WHT $W_{h}(x)$ of $z(k)$ is defined as

$W_{h}(x)=\sum_{k=0}^{N-1} z(k)(-1)^{\sum_{m=0}^{u-1} x_{m} k_{m}}, 0 \leq x \leq N-1$

here $x_{m}$ and $k_{m}, 0 \leq m \leq u-1, x_{m}$ and $k_{m}$ values are in 0 or 1,represent the binary digits of $x$ and $m$,respectively, i.e.,

$x=x_{m-1} 2^{m-1}+x_{m-2} 2^{m-2}+\ldots+x_{2} 2^{2}+x_{1} 2+x_{0}$

$k=k_{m-1} 2^{m-1}+k_{m-2} 2^{m-2}+\ldots+k_{2} 2^{2}+k_{1} 2+k_{0}$

WHT matrix form of (2.1) is

$W_{h}=W_{N} \cdot z$

Where $W_{N}$ represents the WHT matrix

$W_{N}(x, k)=\sum_{m=0}^{u-1} x_{m} k_{m}, 0 \leq m, k \leq u-1$

and inverse form of $(2.3)$ is $W_{N}^{(-1)}=(1 / N) W_{N}$. The matrix $W_{N}$ is derived by Kronecker product using $W_{2}$ matrix

$W_{N}=W_{2} \otimes W_{2} \otimes W_{2} \otimes \ldots \otimes W_{2}$

here $\otimes$ denotes the Kronecker product, $W_{2}=\left[\begin{array}{cc}1 & 1 \\ 1 & -1\end{array}\right]$ and the number of $W_{2}$ used in (2.4) is $u$ value.

For example $\mathrm{N}=8$ then $u=\log _{2} 8=3$ and WHT is

$W_{8}=W_{2} \otimes W_{2} \otimes W_{2}$

$$
W_{8}=\left[\begin{array}{cccccccc}
1 & 1 & 1 & 1 & 1 & 1 & 1 & 1 \\
1 & -1 & 1 & -1 & 1 & -1 & 1 & -1 \\
1 & 1 & -1 & -1 & 1 & 1 & -1 & -1 \\
1 & -1 & -1 & 1 & 1 & -1 & -1 & 1 \\
1 & 1 & 1 & 1 & -1 & -1 & -1 & -1 \\
1 & -1 & 1 & -1 & -1 & 1 & -1 & 1 \\
1 & 1 & -1 & -1 & -1 & -1 & 1 & 1 \\
1 & -1 & -1 & 1 & -1 & 1 & 1 & -1
\end{array}\right]
$$

\section{DCT, Signed DCT and Binary DCT}

Let $z(k)$ be a sequence of length $\mathrm{N}$. The one dimensional $Z_{d c t}(k)$ of $z(k)$ is defined as

$$
Z_{d c t}(k)=a_{p} \sum_{m=0}^{N-1} z(k) \cos \cos \left(\frac{(2 m+1) p \pi}{2 N}\right), 0 \leq p \leq N-1
$$


where $a_{p}=\left\{\begin{array}{l}1, \text { for } m=0 \\ \sqrt{2}, \text { otherwise }\end{array}\right.$

The two dimensional $Z_{\text {dct } 2}(k)$ is

$Z_{d c t 2}(k)=a(p) a(q) \sum_{m=0}^{N-1} \sum_{l=0}^{N-1} z(p, q) \cos \left(\frac{(2 m+1) p \pi}{2 N}\right) \cos \left(\frac{(2 l+1) q \pi}{2 N}\right) 0 \leq p, q \leq N-1$

where $a(t)=\left\{\begin{array}{c}1, \text { for } t=0 \\ \sqrt{2}, \text { otherwise }\end{array}\right.$

The DCT matrix expressed by (3.2) is

$$
Z_{d c t}=X_{N} \cdot k
$$

where $X_{N}$ the DCT matrix is whose entries are given by

$X_{N}(p, k)=a_{p} \cos \left(\frac{(2 m+1) p \pi}{2 N}\right), 0 \leq p, k \leq N-1$

and its inverse is $X_{N}^{-1}=(1 / N) X_{N}^{T}$, with $T$ denoting the transpose operation. For example $\mathrm{N}=8$ the DCT matrix from (3.4).

$$
X_{8}=\left[\begin{array}{llllllll}
x 5 & x 5 & x 5 & x 5 & x 5 & x 5 & x 5 & x 5 \\
x 1 & x 2 & x 3 & x 4 & -x 4 & -x 3 & -x 2 & -x 1 \\
x 6 & x 7 & -x 7 & -x 6 & -x 6 & -x 7 & x 7 & x 6 \\
x 2 & -x 4 & -x 1 & -x 3 & x 3 & x 1 & x 4 & -x 2 \\
x 5 & -x 5 & -x 5 & x 5 & x 5 & -x 5 & -x 5 & x 5 \\
x 3 & -x 1 & x 4 & x 2 & -x 2 & -x 4 & x 1 & -x 3 \\
x 7 & -x 6 & x 6 & -x 7 & -x 7 & x 6 & -x 7 & x 7 \\
x 4 & -x 3 & x 2 & -x 1 & x 1 & -x 2 & x 3 & -x 4
\end{array}\right]
$$

here $\mathrm{x} 1, \mathrm{x} 2, \mathrm{x} 3, \mathrm{x} 4, \mathrm{x} 5, \mathrm{x} 6$ and $\mathrm{x} 7$ are positive numbers, and $\mathrm{x} 1=0.4904, \mathrm{x} 2=0.4157$, $\mathrm{x} 3=0.2778, \mathrm{x} 4=0.0975, \mathrm{x} 5=0.3536, \mathrm{x} 6=0.4619, \mathrm{x} 7=0.1913$, and now find the signed version of (10), here use the signum function for finding the signed DCT matrix. Signum function is as like

$\operatorname{sign}(t)=\left\{\begin{array}{c}+1, \text { if } t>0 \\ 0, \text { if } t=0 \\ -1, \text { if } t<0\end{array}\right.$

$\bar{X}_{8}=\left[\begin{array}{cccccccc}1 & 1 & 1 & 1 & 1 & 1 & 1 & 1 \\ 1 & 1 & 1 & 1 & -1 & -1 & -1 & -1 \\ 1 & 1 & -1 & -1 & -1 & -1 & 1 & 1 \\ 1 & -1 & -1 & -1 & 1 & 1 & 1 & -1 \\ 1 & -1 & -1 & 1 & 1 & -1 & -1 & 1 \\ 1 & -1 & 1 & 1 & -1 & -1 & 1 & -1 \\ 1 & -1 & 1 & -1 & -1 & 1 & -1 & 1 \\ 1 & -1 & 1 & -1 & 1 & -1 & 1 & -1\end{array}\right]$

The (3.6) signed DCT matrix is not an orthogonal matrix, so it is not satisfies the WHT properties that's why we are going to change above matrix into orthogonal. Now compare the results of WHT matrix and signed DCT matrix then all rows signed DCT are approximately equal to any row of WHT, for satisfying WHT properties change some rows of signed DCT matrix with approximate rows of WHT matrix rows. By above theory first, second, third, fifth, seventh and eighth rows of $\bar{X}_{8}$ are equal to one of the row in $W_{8}$, change the rows of signed DCT with minimum elements entry, then the fourth row of $\bar{X}_{8}$ can only replaced by eighth or third rows of $W_{8}$, and the sixth row of $\bar{X}_{8}$ can only replaced 
by eighth. Now fourth row of $\bar{X}_{8}$ can replaced with third row of the $W_{8}$ [1].The binary DCT matrix is as shown in bellow

$\bar{X}_{8}=\left[\begin{array}{cccccccc}1 & 1 & 1 & 1 & 1 & 1 & 1 & 1 \\ 1 & 1 & 1 & 1 & -1 & -1 & -1 & -1 \\ 1 & 1 & -1 & -1 & -1 & -1 & 1 & 1 \\ 1 & 1 & -1 & -1 & 1 & 1 & -1 & -1 \\ 1 & -1 & -1 & 1 & 1 & -1 & -1 & 1 \\ 1 & -1 & -1 & 1 & -1 & 1 & 1 & -1 \\ 1 & -1 & 1 & -1 & -1 & 1 & -1 & 1 \\ 1 & -1 & 1 & -1 & 1 & -1 & 1 & -1\end{array}\right]$

this one for $\mathrm{N}=8$. By applying the above procedure for $\mathrm{N}$ number of elements then the closed form equation is given bellow

$\overline{\bar{X}}_{N}(x, k)=(-1)^{\sum_{l=0}^{n-1}\left(\left(x_{i}+x_{t+1}\right) k_{k-1-1}\right)}, 0 \leq x, k \leq N-1$

This expression getting form different cases are taken for when comparing the WHT and Signed DCT, finally (3.8) for N number of Binary DCT matrix.

\section{DHT and Binary DHT}

Let $z(k)$ be a sequence of length $\mathrm{N}$. The one dimensional DHT $Z_{d h t}(k)$ of $z(k)$ is defined as

$Z_{d h t}(k)=\sum_{m=0}^{N-1} z(m) \operatorname{cas}\left(\frac{2 \pi m k}{N}\right), 0 \leq k \leq N-1$

and two dimensional DHT is defined as

$Z_{d h h_{2}}(k)=\sum_{m=0}^{N-1} \sum_{l=0}^{N-1} z(m, l) \operatorname{cas}\left(\frac{2 \pi m k}{N}\right) \operatorname{cas}\left(\frac{2 \pi l k}{N}\right), 0 \leq k \leq N-1$

where $\operatorname{cas}(x)=\cos (x)+\sin (x)$

The matrix form for (4.1) DHT is

$Z_{d h t}=Y_{h} \cdot k$

the DHT matrix is as shown bellow

$Y_{h}(k, m)=\operatorname{cas}\left(\frac{2 \pi m k}{N}\right), 0 \leq k, m \leq N-1$

$Y_{8}=\left[\begin{array}{cccccccc}1 & 1 & 1 & 1 & 1 & 1 & 1 & 1 \\ 1 & \sqrt{2} & 1 & 0 & -1 & -\sqrt{2} & -1 & 0 \\ 1 & 1 & -1 & -1 & 1 & 1 & -1 & -1 \\ 1 & 0 & -1 & \sqrt{2} & -1 & 0 & 1 & -\sqrt{2} \\ 1 & -1 & 1 & -1 & 1 & -1 & 1 & -1 \\ 1 & -\sqrt{2} & 1 & 0 & -1 & \sqrt{2} & -1 & 0 \\ 1 & -1 & -1 & 1 & 1 & -1 & -1 & 1 \\ 1 & 0 & -1 & -\sqrt{2} & -1 & 0 & 1 & \sqrt{2}\end{array}\right]$

using sigma function finds the signed DHT of (4.5) 


$$
\bar{Y}_{8}=\left[\begin{array}{cccccccc}
1 & 1 & 1 & 1 & 1 & 1 & 1 & 1 \\
1 & 1 & 1 & 0 & -1 & -1 & -1 & 0 \\
1 & 1 & -1 & -1 & 1 & 1 & -1 & -1 \\
1 & 0 & -1 & 1 & -1 & 0 & 1 & -1 \\
1 & -1 & 1 & -1 & 1 & -1 & 1 & -1 \\
1 & -1 & 1 & 0 & -1 & 1 & -1 & 0 \\
1 & -1 & -1 & 1 & 1 & -1 & -1 & 1 \\
1 & 0 & -1 & -1 & -1 & 0 & 1 & 1
\end{array}\right]
$$

The (4.6) signed DHT matrix is not an orthogonal matrix, so it is not satisfies the WHT properties that's way we are going to change above matrix into orthogonal. Now compare the results of WHT matrix and signed DHT matrix then all rows signed DHT are approximately equal to any row of WHT, for satisfying WHT properties change some rows of signed DHT matrix with approximate rows of WHT matrix rows. By this procedure the Binary DHT matrix for $\mathrm{N}=8$ as shown in bellow

$$
\overline{Y_{8}}=\left[\begin{array}{cccccccc}
1 & 1 & 1 & 1 & 1 & 1 & 1 & 1 \\
1 & 1 & 1 & 1 & -1 & -1 & -1 & -1 \\
1 & 1 & -1 & -1 & 1 & 1 & -1 & -1 \\
1 & -1 & -1 & 1 & -1 & 1 & 1 & -1 \\
1 & -1 & 1 & -1 & 1 & -1 & 1 & -1 \\
1 & -1 & 1 & -1 & -1 & 1 & -1 & 1 \\
1 & -1 & -1 & 1 & 1 & -1 & -1 & 1 \\
1 & 1 & -1 & -1 & -1 & -1 & 1 & 1
\end{array}\right]
$$

this one for $\mathrm{N}=8$. By applying the above procedure for $\mathrm{N}$ number of elements then the closed form equation is given bellow

$\overline{\bar{Y}}_{8}(x, k)=(-1)^{\sum_{t=0}^{n-1}\left(\left(s_{u-1-t}+s_{u-2-t}\right) k_{t}\right)}, 0 \leq x, k \leq N-1$

here $s_{i}, 0 \leq t \leq u-1$, are the binary digits of $s_{s=(N-k) \bmod N}$ with $s_{-1}=0$, This expression getting form different cases are taken for when comparing the WHT and Signed DHT [1], finally (4.8) for N number of Binary DHT matrix.

\section{Haar Transform and Binary Haar Transform}

The Harr function $h_{k}(z)$, which are defined in the closed interval [0,1]. The order $\mathrm{k}$ of the function is uniquely decomposed into two elements $\mathrm{i}, \mathrm{j}$ [12].

$k=2^{i}=j-1, k=0,1, \ldots, N-1$, and $N=2^{n}$

Where $0 \leq p \leq N-1,0 \leq j \leq 2^{i}$ for $i \neq 0$ and $j=0$ or 1 for $i=0$

The Haar transform is

$$
h_{k}(z)=h_{i j}(z)=\frac{1}{\sqrt{N}}\left\{\begin{array}{c}
2^{i / 2} \frac{j-1}{2^{i}} \leq z \leq \frac{j-1}{2^{i}} \\
-2^{i / 2} \frac{j-1}{2^{i}} \leq z \leq \frac{j}{2^{i}} \\
0 \text { otherwise }[0,1]
\end{array}\right.
$$

for $\mathrm{N}=8$ the Haar transform matrix is 


$$
H=\left[\begin{array}{cccccccc}
1 & 1 & 1 & 1 & 1 & 1 & 1 & 1 \\
1 & 1 & 1 & 1 & -1 & -1 & -1 & -1 \\
\sqrt{2} & \sqrt{2} & -\sqrt{2} & -\sqrt{2} & 0 & 0 & 0 & 0 \\
0 & 0 & 0 & 0 & \sqrt{2} & \sqrt{2} & -\sqrt{2} & -\sqrt{2} \\
2 & -2 & 0 & 0 & 0 & 0 & 0 & 0 \\
0 & 0 & 2 & -2 & 0 & 0 & 0 & 0 \\
0 & 0 & 0 & 0 & 2 & -2 & 0 & 0 \\
0 & 0 & 0 & 0 & 0 & 0 & 2 & -2
\end{array}\right]
$$

for Signed Haar Transform matrix we applied a signum function to (5.3)

$$
\bar{H}=\left[\begin{array}{cccccccc}
1 & 1 & 1 & 1 & 1 & 1 & 1 & 1 \\
1 & 1 & 1 & 1 & -1 & -1 & -1 & -1 \\
1 & 1 & -1 & -1 & 0 & 0 & 0 & 0 \\
0 & 0 & 0 & 0 & 1 & 1 & -1 & -1 \\
1 & -1 & 0 & 0 & 0 & 0 & 0 & 0 \\
0 & 0 & 1 & -1 & 0 & 0 & 0 & 0 \\
0 & 0 & 0 & 0 & 1 & -1 & 0 & 0 \\
0 & 0 & 0 & 0 & 0 & 0 & 1 & -1
\end{array}\right]
$$

Now compare the results of WHT matrix and signed Haar Transform matrix then all rows signed Harr Transform are approximately equal to any row of WHT, for satisfying WHT properties change some rows of signed Haar Transform matrix with approximate rows of WHT matrix rows [1]. By this procedure the Binary Haar Transform matrix for $\mathrm{N}=8$ as shown in bellow

$$
\vec{H}=\left[\begin{array}{cccccccc}
1 & 1 & 1 & 1 & 1 & 1 & 1 & 1 \\
1 & 1 & 1 & 1 & -1 & -1 & -1 & -1 \\
1 & 1 & -1 & -1 & -1 & -1 & 1 & 1 \\
1 & 1 & -1 & -1 & 1 & 1 & -1 & -1 \\
1 & -1 & 1 & -1 & -1 & 1 & -1 & 1 \\
1 & -1 & 1 & -1 & 1 & -1 & 1 & -1 \\
1 & -1 & -1 & 1 & 1 & -1 & -1 & 1 \\
1 & -1 & -1 & 1 & -1 & 1 & 1 & -1
\end{array}\right]
$$

by applying the above procedure to the Haar Transform and WHT matrices of higher orders of $\mathrm{N}$ and studying various cases separately to find a closed form expression for the elements of the corresponding matrix in terms of the indices of their locations, a Binary Haar Transform matrix of any order $\mathrm{N}$ can be written as

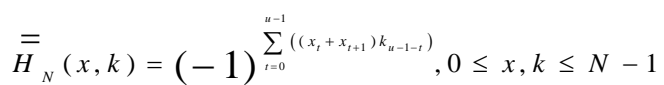

and necessary conditions for getting Binary Haar Transform matrix swap the $(\mathrm{N}-3)^{\text {th }}$ row with $(\mathrm{N}-1)^{\text {th }}$ and $(\mathrm{N}-2)^{\text {th }}$ row with $\mathrm{N}^{\text {th }}$ row of $\overline{\bar{H}}_{N}$ after that getting a Binary Haar transform matrix, for $\mathrm{N}$ number of sequence DCT computation requires $\mathrm{N}^{2}$ multiplications and $\mathrm{N}(\mathrm{N}$ 1) additions and Haar transform computational requires $N$ number of multiplications and 2(N-1) numbers of additions, for Binary DCT and Binary Haar Transformation requires $N \log _{2}(N)$ additions.

\section{Image Compression}

The following is a general way of image compression process. 
1. Image is broken into 8 by 8 blocks of pixels.

2. Working from left to right and top to bottom, the DCT, Signed DCT, Binary DCT, Haar Transform and Binary Haar Transforms are applied to each block.

3. Each block compressed through a mask matrix.

4. The array of compressed blocks that constitute the image is stored in a drastically reduced amount of space.

5. When desired, the image is reconstructed through decompression, a process that uses the inverse of DCT, Signed DCT, Binary DCT, Haar Transform and Binary Haar Transforms.

The DCT, Binary DCT, Haar Transform and Binary Haar Transforms matrices satisfy the property of orthogonality [4], i.e., inverse of matrix is equal to the transpose matrix or multiplication of matrix and transpose matrix is equal to identity matrix. The Signed DCT is not satisfying the orthogonal property then derives the corresponding inverse matrix for the decompression process. Let $\mathrm{Z}$ be an 8 by 8 block of image data and $\mathrm{X}$ be its corresponding matrix in transformed domain. Then the transformed operation will be $X=T Z T^{\prime}$. Since $\mathrm{T}$ is orthogonal, now reconstruct the image by using the reverse transform given as $Z=T X T^{\prime}$. Where the $\mathrm{T}$ matrix for DCT, Signed DCT, Binary DCT, Haar Transform and Binary Haar Transforms represents the $X_{8}, \bar{X}_{8}, \overline{\bar{X}}_{8}, \bar{H}_{8}$ and $\overline{\bar{H}}_{8}$ respectively.

The results for image compression and decompression using DCT, Signed DCT, Binary DCT, Haar Transform and Binary Haar Transforms are illustrated in Figures 2(a)-2(h), and Table 1. From Table 1, it can be seen that Binary DCT and Binary Haar Transform gives the better results.

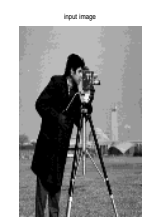

\section{Figure 1. Input Image}
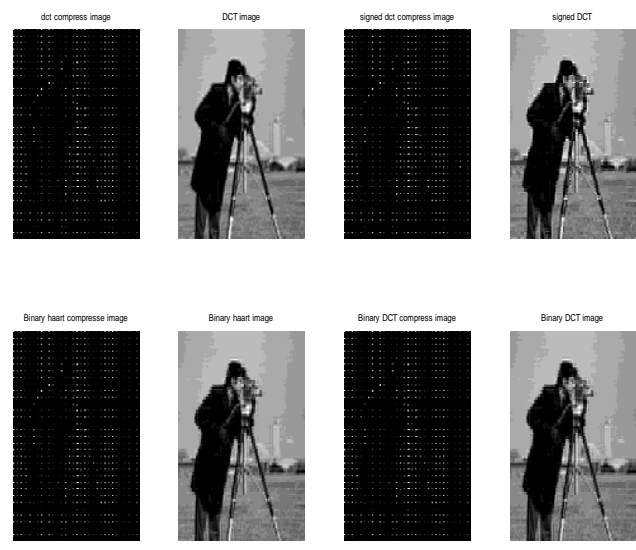

Figure 2. (a) Compressed Image Using DCT (b) Decompressed Image Using DCT, (c) Compressed Using Signed DCT (d) Decompressed Image Using Signed DCT, (e) Compressed Using Binary DCT (f) Decompressed Image Using Binary DCT, (f) Compressed Using Binary Haar Transform (h) Decompressed Image Using Binary Haar Transform 
The results of image compression and decompression image sizes and PSNR values.

Table 1. Size and PSNR Values of Image Using Different Techniques

\begin{tabular}{|c|c|c|c|c|}
\hline $\begin{array}{c}\text { Transfo } \\
\text { rms }\end{array}$ & $\begin{array}{c}\text { Input } \\
\text { image } \\
\text { size(K } \\
\text { B) }\end{array}$ & $\begin{array}{c}\text { Compre } \\
\text { ssed } \\
\text { image } \\
\text { size(KB } \\
\text { ) }\end{array}$ & $\begin{array}{c}\text { Decompre } \\
\text { ssed image } \\
\text { size(KB) }\end{array}$ & $\begin{array}{c}\text { PSNR } \\
\text { (in dB) }\end{array}$ \\
\hline DCT & 104 & 77 & 483 & $\begin{array}{c}71.954 \\
5\end{array}$ \\
\hline $\begin{array}{c}\text { Signed } \\
\text { DCT }\end{array}$ & 104 & 68 & 269 & $\begin{array}{c}71.362 \\
2\end{array}$ \\
\hline $\begin{array}{c}\text { Binary } \\
\text { DCT }\end{array}$ & 104 & 58 & 105 & $\begin{array}{c}71.208 \\
8\end{array}$ \\
\hline $\begin{array}{c}\text { Binary } \\
\text { Haar } \\
\text { Transfo } \\
\text { rm }\end{array}$ & 104 & 57.4 & 105 & $\begin{array}{c}71.208 \\
8\end{array}$ \\
\hline
\end{tabular}

\section{ECG Data Compression}

Steps for ECG Data Compression:

1. The input image is converted from color image to grey scale image and then the grey scale image is converted to a square matrix. Image having pixels 512 by 512 .

2. DCT, Signed DCT and Binary DCT transform techniques are applied for data compression and decompression [8].

Figure 3, show the results of ECG data compression and decompression
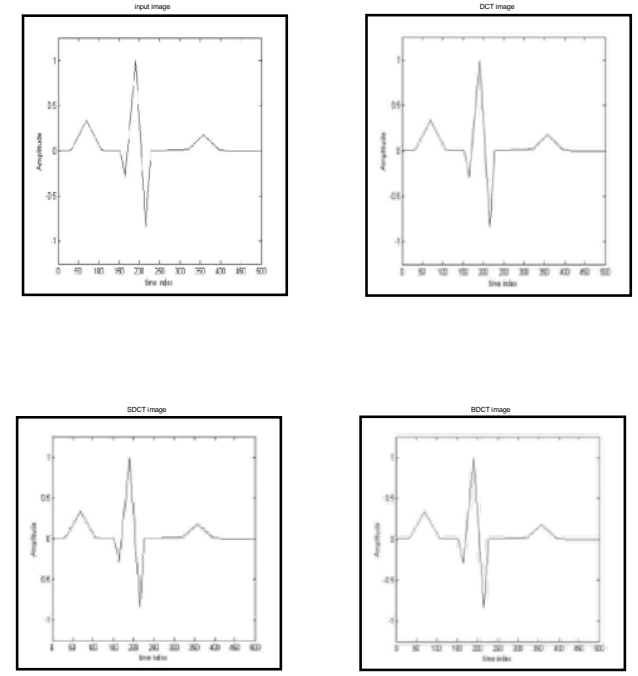

Figure 3. (a) Input Image and Decompression of ECG Data Using DCT Technique (b) Decompression of ECG Data Using Signed DCT Technique and Binary DCT Technique

Mean square values of ECG 512 using DCT, Signed DCT and Binary DCT are 0.0051, .0066 and 0.0059 respectively Binary DCT is preferred it uses only additions to reduce the arithmetic complexity. 


\section{Image Encryption}

Image encryption is a well known technique in recent communication networks to prevent any non authorized utilization or modification of the exchange images and videos. Double random phase encoding technique is well known for image encryption, which employs complex valued Discrete Fourier Transform. The double random phase encoding has been modified to introduce the double random amplitude encoding to deal with real valued sequences using real valued transforms such as DHT [3].

Figure 4 , illustrates the proposed encryption process. It should be noted that $\mathrm{a}(\mathrm{x}, \mathrm{y})$ and $\mathrm{b}(\mathrm{x}, \mathrm{y})$ are two $\mathrm{N}$ by $\mathrm{N}$ random amplitude matrices, these are distributed between $[-1+1]$ and independent of each other [1]. The equation for image encryption and decryption using random amplitude keys are

$U=\left(1 / N^{2}\right) \overline{\bar{Y}}_{N}^{\prime}\left(\left(\overline{\bar{Y}}_{N}(X \square[a(x, y)]) \overline{\bar{Y}}_{N}^{\prime}\right) \square[b(x, y)]\right) \overline{\bar{Y}}_{N}$,

$\mathrm{U}$ refers the encrypted image, $\mathrm{X}$ is the input image data and $\overline{\bar{Y}}_{N}$ is the transform matrix. Where $\square$ denotes the element by element multiplication.

$V=\left(1 / N^{2}\right)\left(\overline{\bar{Y}}_{N}{ }^{\prime}\left(\left(\overline{\bar{Y}}_{N} U \overline{\bar{Y}}_{N}\right) \varnothing[\hat{a}(x, y)]\right) \overline{\bar{Y}}_{N}\right) \varnothing[\hat{b}(x, y)]$,

$\mathrm{V}$ refers the decrypted image or reconstructed image, $a(x, y)=\hat{a}(x, y)$ and $b(x, y)=b(x, y)$.
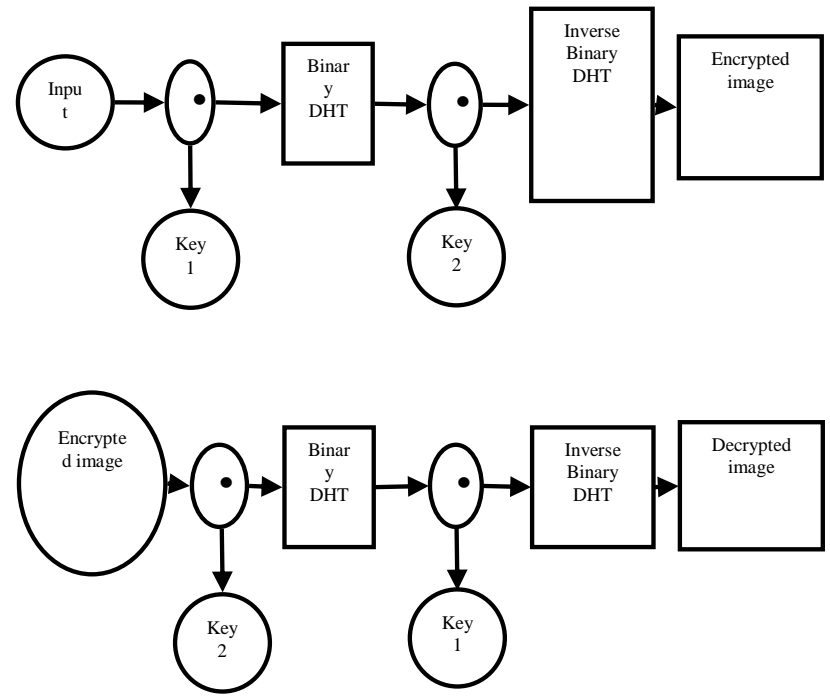

Figure 4. (a) Proposed Encryption Process Using Binary DHT, (b) Proposed Decrypted Process Using Binary DHT

Figures 5(a)-5(g), illustrate the encryption and decryption of an input image using random amplitude keys. 


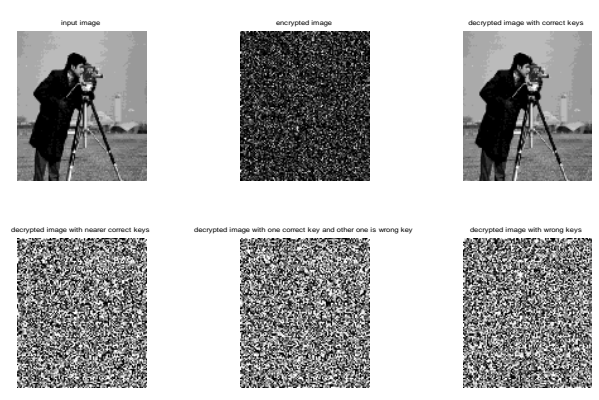

Figure 6: (a) Input Image, (b) Encrypted Image, (c) Decrypted Image with Correct Keys, (d) Decrypted with Nearest Correct Keys, (e) Decrypted with One Correct Key, One Wrong Key, (f) Decrypted with Both are Wrong Keys

\section{Conclusion}

The image compression using DCT, Signe DCT and Binary DCT were implemented and it was observed that Signed DCT technique could not give better results since it was not satisfying WHT properties. Out of the three techniques Binary DCT and Binary Haar transform gave better results.

For ECG data compression and decompression the same techniques were used. Though the mean square error values are nearly equal DCT was found to be better technique since it only uses additions and less complexity.

The image encryption and decryption using Binary DHT technique proved that it is robust technique which uses random amplitude keys and unless keys are perfectly matched the decryption is not possible.

\section{References}

[1] S. Bouguezel, M. O. Ahmad, Fellow, IEEE, and M. N. S. Swamy, Fellow, IEEE transactions on circuits and systems-i: regular papers, vol. 60, no. 4, (2013) April.

[2] R. K. Senapati, U. C. Pati and K. K Mahapatra, "An Efficient Sparse $8 \times 8$ Orthogonal Transform Matrix for Color Image Compression", (2007) April.

[3] L. Chen and D. Zhao, "Optical image encryption with Hartley transforms", Opt. Lett., vol. 31, (2006) Dec., pp. 3438-3440.

[4] G. K. Wallace, "The JPEG still picture compression standard", Commun. ACM, vol. 34, (1991), pp. 30 44.

[5] C. H. Paik and M. D. Fox, "Fast Hartley transform for image processing", IEEE Trans. Med. Imag., vol. 7, no. 6, (1988) Jun., pp. 149-153.

[6] K. J. Horadam, "Hadamard Matrices and Their Applications", Princeton, NJ: Princeton Univ. Press, (2006).

[7] M. Puschel, "Cooley-Tukey FFT like algorithms for the DCT", in Proc.IEEE ICASSP'03, vol. 2, (2003) Apr., pp. 501-504.

[8] Er. A. Sayeed, "ECG Data Compression Using DWT \& HYBRID", vol. 3, no. 1, (2013) January February, pp. 422-425 422.

[9] D. J. L. Gall, "MPEG: A video compression standard for multimedia applications", Commun. ACM, vol. 34, (1991) Apr., pp. 47-58.

[10] N. Ahmed, T. Natarajan and K. R. Rao, "Discrete cosine transform”, IEEE Trans. Comput., vol. C-23, no. 1, (1974) Jan., pp. 90-93.

[11] I. Valova and Y. Kosugi, "Hadamard-Based Image Decomposition and compression", IEEE transactions on information technology in biomedicine, vol. 4, no. 4, (2000) December.

[12] R. S. Stankovic and B. J. Falkowski, "The Haar wavelet transform: its status and achievements", Computers and Electrical Engineering, vol. 29, (2003), pp. 25-44. 